\title{
Quantum Electrostatic Shock-Waves in Symmetric Pair-Plasmas
}

\author{
Massoud Akbari-Moghanjoughi \\ Department of Physics, Faculty of Sciences, Azarbaijan University of Tarbiat Moallem, Tabriz, Iran \\ Email: m_akbari@azaruniv.edu
}

Received February 16, 2012; revised March 15, 2012; accepted March 23, 2012

\begin{abstract}
In this paper, the quantum hydrodynamics (QHD) model is used to study the propagation of small- but finite-amplitude quantum electrostatic shock-wave in an inertial-less symmetric pair (ion) plasma with immobile background positive constituents. The dispersion due to the quantum tunneling and inertial effects as well as dissipation caused by particle collisions leading to the shock-like or double-layer structures are considered. Investigation of both the stationary and traveling-wave solutions to Kortewege-de Veries-Burgers evolution equation show that critical values exist which govern the type of collective plasma structures. Current analysis apply to diverse kind of symmetric plasmas such as laboratory inertially confined or astrophysical pair-ion or electron-positron degenerate plasmas.
\end{abstract}

Keywords: Electrostatic Shock-Wave; Quantum Plasma; Fermi Statistics; Quantum Hydrodynamics; KdVB Equation; QHD

\section{Introduction}

Owing to the wide applicability in miniaturization techniques in electronic devices, quantum plasma has received a great deal of attention in recent years, both from theoretical and experimental points of view [1-3]. Much of understanding of the properties of degenerate ionized matter under the name quantum plasma is inspired by the pioneering works of Bohm, Pines and Levine [4-6]. It is well-known that the quantum electronic transport effects play inevitable role in metallic and semiconductor matterials as well as newly introduced concepts called nanostructured compounds such as quantum-wells, wires and dots [7] in which the electrons and holes can be regarded as quantum degenerate ingredients [8]. Quantum effects also play crucial role in nonlinear processes in compact astrophysical objects, such as white-dwarfs, neutron stars and pulsars etc. [9]. It has been pointed out [2] that compact objects such as white dwarfs, in which the electron number-density is much higher reaching for instance $10^{30}$ $\mathrm{cm}^{-1}$ many orders of magnitude larger than that in ordinary metals, can be considered as hot fusion-like plasma. However, these objects still behave as cold completely degenerate quantum system called the Fermi gas. It has been shown that, in a degenerate electron-positron-ion plasma, unlike common sense, the annihilation rates can be much lower than that expected and this leaves enough time to consider the nonlinear effects in these plasmas. In quantum plasmas unlike the classical counterparts only the electrons/positrons with the energies within the small range around the Fermi-energy take part in collective plasma processes. Recent evaluations [10-12] show that the annihilation criteria can be relaxed and that in white dwarfs, for instance, the positrons are long lived enough to contribute to effective nonlinear phenomena.

On the other hand, the creation of dense fullerene-pair plasma in laboratory has recently been reported [13-15] and verity of nonlinear waves in such environment has been confirmed experimentally. However, the addition of other charged particles such as dust particulates into pairion plasma in order to produce three component plasmas accommodating different types of nonlinear wave phenomena may also be anticipated. Essentially, there are many mathematical formulations to describe the linear and nonlinear properties of charged particles at quantum levels in a dense degenerate plasma. The quantum hydrodynamics (QHD) model, which is based on the Schrödinger-Poisson formulations [2], has an advantage of being related to thermodynamic properties of system under investigation with the main drawback in this approach which is its deficiency in describing the Landau damping [16]. QHD model has been recently used to investigate the wave-dynamics in diverse plasma systems [17-26].

Furthermore, unlike classical fluids, quantum plasma exhibits dispersion instead of dissipation, which is caused by the quantum tunneling effects associated with the Bohm-potential [27]. However, dissipation may arise due 
to the kinematic viscosity and collisions, thus, the wave propagation is governed by delicate interplay between the quantum tunneling and wave-particle interactions. In such dissipative plasma medium the propagation of smallbut finite-amplitude nonlinear excitations may be adequately described by the Korteweg-de Vries-Burgers (KdVB) equation. In this study the QHD model is applied to study the nonlinear dynamics of electrostatic (ES) shock waves in three component pair-plasma. Many authors has recently applied the QHD model to study the ion-acoustic and dust-acoustic shock-wave dynamics in both non-relativistic [28-31] and relativistic [32] electron-positron-ion plasma in planar or non-planar geometries. However, electron-positron-ion plasma is only a special case of pair-ion plasma in which simplifications can be made in QHD equation due to the small electronto-ion mass ratio. In this study we use a more general approach including the inertia of degenerate particles which can be readily applied to an arbitrary mass pair-ion plasmas.

The organization of the article is as follows. Description of basic quantum plasma equations is given in Section 2. Reductive perturbation method is applied and the $\mathrm{KdVB}$ evolution equation is obtained in Section 3. In Section 4 the numerical findings and discussions based on the numerical analysis are presented. Finally, Section 5 devotes to the concluding remarks and summary.

\section{Description of Quantum Plasma State}

Consider a dense plasma of degenerate fermion gas of pair-ion or electron-positron in the presence of heavy background inertial positive ions. A one dimensional QHD plasma-model is described by continuity and momentum equations in addition to the Poisson's relation. However, in this QHD-model we also use the degeneracy pressure caused by Pauli exclusion rule and the quantum tunneling due to Bohm-force, a consequence of wave-like nature in quantum physics. Therefore, ignoring the classical pressure due to immobile ingredients a complete set of equations [33] describing the dynamics of this degenerate plasma, may be written in dimensional form as

$$
\begin{aligned}
\frac{\partial n_{+}}{\partial t}+\frac{\partial u_{+} n_{+}}{\partial x}= & 0, \\
\frac{\partial n_{-}}{\partial t}+\frac{\partial u_{-} n_{-}}{\partial x}= & 0, \\
\frac{\partial u_{+}}{\partial t}+u_{+} \frac{\partial u_{+}}{\partial x}= & -\frac{Z_{+}}{m_{+}} \frac{\partial \phi}{\partial x}-\frac{1}{m_{+} n_{+}} \frac{\partial p_{+}}{\partial x}+\mu_{+} \frac{\partial^{2} u_{+}}{\partial x^{2}} \\
& +\frac{\hbar^{2}}{2 m_{+}^{2}} \frac{\partial}{\partial x}\left[\frac{1}{\sqrt{n_{+}}} \frac{\partial^{2} \sqrt{n_{+}}}{\partial x^{2}}\right],
\end{aligned}
$$

$$
\begin{aligned}
& \frac{\partial u_{-}}{\partial t}+u_{-} \frac{\partial u_{-}}{\partial x}= \frac{Z_{-}}{m_{-}} \frac{\partial \phi}{\partial x}-\frac{1}{m_{-} n_{-}} \frac{\partial p_{-}}{\partial x}+\mu_{-} \frac{\partial^{2} u_{-}}{\partial x^{2}} \\
&+\frac{\hbar^{2}}{2 m_{-}^{2}} \frac{\partial}{\partial x}\left[\frac{1}{\sqrt{n_{-}}} \frac{\partial^{2} \sqrt{n_{-}}}{\partial x^{2}}\right], \\
& \frac{\partial^{2} \phi}{\partial x^{2}}=\frac{e}{\varepsilon_{0}}\left(Z_{p}\left(n_{-}-n_{+}\right)-Z_{h} N_{h}\right)
\end{aligned}
$$

where, the \pm signs differentiate among pair charges. The quantities $\mu_{ \pm}$and $\hbar$ are kinematic viscosities of plasma and the scaled Plank constant, respectively. Also, the subscripts $p$ and $h$ refer to pair and heavy constituents, correspondingly. Furthermore, we will assume $Z_{p}=1$ for simplicity. It is noticed that, in a quantum plasmaunder the zero-temperature Fermi-gas assumption (with the thermal temperatures being negligible compared to the Fermi-temperatures), the non-relativistic degeneracy pressure is ruled by Pauli exclusion principle and relates to the particle quantum number-density through the following relation

$$
p_{ \pm}=\frac{m_{p} v_{F \pm}^{2}}{3 n_{ \pm, 0}^{2}} n_{ \pm}^{3}, \quad v_{F \pm}=\sqrt{\frac{2 E_{F \pm}}{m_{p}}}, \quad E_{F \pm}=k_{B} T_{F \pm} .
$$

Here, the quantities $v_{F \pm}, E_{F \pm}$ and $E_{F \pm}$ denote Fermi-velocity, Fermi-energy and Fermi-temperature, respectively and $n_{ \pm, 0}$ represents the pair equilibrium number-density. On the other hand, by standard definitions it is known that, in a one-dimensional degenerate Fermi-gas the equilibrium quantum number-densities are related to Fermi-temperature via

$$
T_{F \pm}=\frac{\left(3 \pi^{2} \hbar n_{ \pm, 0}\right)^{2}}{2 m_{p} k_{B}} .
$$

In the zero-temperature Fermi-gas the collisions are limited due to a process called Fermi-blocking, hence, such systems may be nearly considered as collision-less. This is fairly valid for dense matter in which the Fermitemperature is well above room-temperature. One should note that, in the fully degenerate plasma regime the socalled de Broglie thermal wavelength

$\lambda_{B}=h /\left(2 \pi m k_{B} T\right)^{1 / 2}$ of particles is equal to or larger than the average inter-particle distance $n^{-1 / 3}$, hence, leading to quantum mechanical effects. However, the wave-particle interactions can be present leading to dissipative effects. Furthermore, the dimension-less QHD equation-set may be obtained using the following scalings

$$
\begin{aligned}
& x \rightarrow \frac{C_{s-}}{\omega_{p-}} x, t \rightarrow \frac{t}{\omega_{p-}}, n \rightarrow n n_{-, 0}, u \rightarrow u C_{s-}, \\
& \varphi \rightarrow \varphi \frac{2 k_{B} T_{F-}}{e} .
\end{aligned}
$$


where, $\omega_{p-}=\sqrt{e^{2} n_{-, 0} / \varepsilon_{0} m_{p}}$ and $C_{s-}=\sqrt{2 k_{B} T_{F-} / m_{p}}$ are the characteristic plasma frequency and Fermi-speed, respectively. Therefore, the normalized compact set of degenerate plasma equations, assuming equal mass and charges for paired particles, becomes

$$
\begin{aligned}
& \frac{\partial n_{\alpha}}{\partial t}+\frac{\partial u_{\alpha} n_{\alpha}}{\partial x}=0 \\
& \frac{\partial u_{\alpha}}{\partial t}+u_{\alpha} \frac{\partial u_{\alpha}}{\partial x}+S_{\alpha} \frac{\partial \phi}{\partial x}+n_{\alpha} \frac{\partial n_{\alpha}}{\partial x}-\eta_{\alpha} \frac{\partial^{2} u_{\alpha}}{\partial x^{2}} \\
& -\frac{H^{2}}{2} \frac{\partial}{\partial x}\left[\frac{1}{\sqrt{n_{\alpha}}} \frac{\partial^{2} \sqrt{n_{\alpha}}}{\partial x^{2}}\right]=0, \\
& \frac{\partial^{2} \phi}{\partial x^{2}}=-\sum_{\alpha} S_{\alpha} n_{\alpha}-Z_{h} N_{h},
\end{aligned}
$$

where, $\alpha=\{+,-\}$ variable denotes the particle chargetype and $S_{\alpha}$ can take the values of $S_{\alpha}=\{+1,-1\}$ for the respective charges and we have $\eta_{\alpha}=\mu_{\alpha} \omega_{p,-} / C_{s,-}$. Moreover, the quantities $u_{\alpha}, n_{\alpha}$ and $\phi$ conventionally refer to the velocity, density of $\alpha$-charged particles and the electrostatic potential, respectively. A normalized purely quantum parameter $H=\hbar \omega_{p-} / 2 k_{B} T_{F-}$, known as quantum diffraction parameter, is introduced above is the ratio of plasmon-energy to the Fermi-energy of the paired particles. It is arguable, that in the $H \rightarrow 0$ limit the classical case should be retained, however, this is not the case since in fact $H$ is proportional to $1 / \hbar$ as it is easily confirmed by Equation (3). Also, in defining the QHD-model we have made use of the degeneracy pressure definition (number-density) instead of the classical one.

The quasi-neutrality condition, on the other hand, is defined through the Poisson's relation at the thermodynamic equilibrium state in the last equation in Equation (5), i.e.

$$
n_{-, 0}-n_{+, 0}-Z N=0,
$$

or equivalently

$$
\beta+\delta=1, \quad \beta=\frac{n_{+, 0}}{n_{-, 0}}, \delta=\frac{Z N}{n_{-, 0}},
$$

where, $Z_{h}$ and $N_{h}$ are the atomic number and density of positive background ions.

\section{Perturbation and KdVB Solution}

Assuming a wave-like perturbation moving with a phasespeed $\lambda$, to find stationary solutions, we can use stretching technique with new variables defined as below

$$
\begin{aligned}
\xi & =\varepsilon^{\frac{1}{2}}(x-\lambda t), \\
\tau & =\varepsilon^{\frac{3}{2}} t .
\end{aligned}
$$

The corresponding reduced state-equations introduced into the new coordinate will be in the following forms

$$
\begin{aligned}
& \varepsilon \frac{\partial n_{\alpha}}{\partial \tau}-\lambda \frac{\partial n_{\alpha}}{\partial \xi}+\frac{\partial u_{\alpha} n_{\alpha}}{\partial \xi}=0, \\
& \varepsilon n_{\alpha}^{3} \frac{\partial u_{\alpha}}{\partial \tau}-\lambda n_{\alpha}^{3} \frac{\partial u_{\alpha}}{\partial \xi}+n_{\alpha}^{3} u_{\alpha} \frac{\partial u_{\alpha}}{\partial \xi} \\
& +S_{\alpha} n_{\alpha}^{3} \frac{\partial \phi}{\partial \xi}+n_{\alpha}^{4} \frac{\partial n_{\alpha}}{\partial \xi}-\varepsilon \eta_{\alpha} n_{\alpha}^{3} \frac{\partial^{2} u_{\alpha}}{\partial x^{2}} \\
& -\varepsilon \frac{H^{2}}{4}\left[\left(\frac{\partial n_{\alpha}}{\partial \xi}\right)^{3}-2 n_{\alpha} \frac{\partial n_{\alpha}}{\partial \xi} \frac{\partial^{2} n_{\alpha}}{\partial \xi^{2}}+n_{\alpha}^{2} \frac{\partial^{3} n_{\alpha}}{\partial \xi^{3}}\right]=0, \\
& \varepsilon \frac{\partial^{2} \phi}{\partial x^{2}}=-\sum_{\alpha} S_{\alpha} n_{\alpha}-Z N .
\end{aligned}
$$

Here, the smallness parameter $\varepsilon$, characterizes the nonlinearity strength and is a very small positive and real number proportional to the perturbation amplitude. Consequently, the asymptotic expansion of plasma variables away from thermodynamics equilibrium can be done using the following orderings [34]

$$
\begin{aligned}
& n_{\alpha}=n_{\alpha, 0}+\varepsilon n_{\alpha}^{(1)}+\varepsilon^{2} n_{\alpha}^{(2)}+\cdots, \\
& u_{\alpha}=\varepsilon u_{\alpha}^{(1)}+\varepsilon^{2} u_{\alpha}^{(2)}+\varepsilon^{3} u_{\alpha}^{(3)}+\cdots, \\
& \phi=\varepsilon \phi^{(1)}+\varepsilon^{2} \phi^{(2)}+\varepsilon^{3} \phi^{(3)}+\cdots, \\
& \eta_{\alpha}=\varepsilon^{\frac{1}{2}} \eta_{0}
\end{aligned}
$$

where, we have used $\eta_{+, 0}=\eta_{-, 0}=\eta_{0}$ due to symmetry and for further simplicity. By applying the orderings defined in Equation (10) in Equation (9) and isolating distinct perturbation-orders, in the leading approximation, we get the following relations through plasma quantities

$$
\begin{aligned}
& \lambda \frac{\partial n_{\alpha}^{(1)}}{\partial \xi}+n_{\alpha, 0} \frac{\partial u_{\alpha}^{(1)}}{\partial \xi}=0, \\
& \lambda \frac{\partial u_{\alpha}^{(1)}}{\partial \xi}+S_{\alpha} \frac{\partial \phi^{(1)}}{\partial \xi}+n_{\alpha}^{(1)} \frac{\partial n_{\alpha}^{(1)}}{\partial \xi}=0, \\
& -\sum_{\alpha} S_{\alpha} n_{\alpha}^{(1)}=0,
\end{aligned}
$$

consequently, the first-order perturbed dynamical plasma components read as

$$
\begin{aligned}
& u_{\alpha}^{(1)}=U_{1 \alpha} \phi^{(1)}, \quad U_{1 \alpha}=\frac{\lambda S_{\alpha}}{\lambda^{2}-n_{\alpha, 0}^{2}}, \\
& n_{\alpha}^{(1)}=N_{1 \alpha} \phi^{(1)}, \quad N_{1 \alpha}=\frac{n_{\alpha, 0} S_{\alpha}}{\lambda^{2}-n_{\alpha, 0}^{2}}, \\
& n_{+}^{(1)}=n_{-}^{(1)} .
\end{aligned}
$$


In such perturbed plasma approximation the nonlinear dispersion relation is also obtained, which in a compact form reads as

$$
\sum_{\alpha} \frac{n_{\alpha, 0}}{\lambda^{2}-n_{\alpha, 0}^{2}}=0
$$

Linear dispersion limits of the electrostatic excitations, in dissipation-less approximation, can be found in Ref. [10]. The compatibility requirement leads to the phasespeed of waves in terms of fractional particles density

$$
\lambda^{2}=\beta \text {. }
$$

Following the same route in perturbation technique, from the next higher-order we get similar relations in neat level of approximation, i.e.

$$
\begin{aligned}
& \frac{\partial n_{\alpha}^{(1)}}{\partial \tau}-\lambda \frac{\partial n_{\alpha}^{(2)}}{\partial \xi}+n_{\alpha, 0} \frac{\partial u_{\alpha}^{(2)}}{\partial \xi}+\frac{\partial u_{\alpha}^{(1)} n_{\alpha}^{(1)}}{\partial \xi}=0 \\
& n_{\alpha, 0}^{3} \frac{\partial u_{\alpha}^{(1)}}{\partial \tau}-\lambda n_{\alpha, 0}^{3} \frac{\partial u_{\alpha}^{(2)}}{\partial \xi}-3 \lambda n_{\alpha, 0}^{2} n_{\alpha}^{(1)} \frac{\partial u_{\alpha}^{(1)}}{\partial \xi} \\
& \quad+n_{\alpha, 0}^{3} u_{\alpha}^{(1)} \frac{\partial u_{\alpha}^{(1)}}{\partial \xi}+3 S_{\alpha} n_{\alpha, 0}^{2} n_{\alpha}^{(1)} \frac{\partial \phi^{(1)}}{\partial \xi}+S_{\alpha} n_{\alpha, 0}^{3} \frac{\partial \phi^{(2)}}{\partial \xi} \\
& \quad+4 n_{\alpha, 0}^{3} n_{\alpha}^{(1)} \frac{\partial n_{\alpha}^{(1)}}{\partial \xi}+n_{\alpha, 0}^{4} \frac{\partial n_{\alpha}^{(2)}}{\partial \xi}-\eta_{0} n_{\alpha, 0}^{3} \frac{\partial^{2} u_{\alpha}}{\partial x^{2}} \\
& \quad-\frac{H^{2}}{4} n_{\alpha, 0}^{2} \frac{\partial^{3} n_{\alpha}^{(1)}}{\partial \xi^{3}}=0, \\
& \frac{\partial^{2} \phi^{(1)}}{\partial \xi^{2}}=-\sum_{\alpha} S_{\alpha} n_{\alpha}^{(2)} .
\end{aligned}
$$

By making use of dispersion relation to eliminate the common terms from Equation (15), we may obtain the $\mathrm{KdVB}$ evolution equation describing the first-order nonlinear evolution of the shock-like nonlinear wave amplitude

$$
\frac{\partial \phi^{(1)}}{\partial \tau}+A \phi^{(1)} \frac{\partial \phi^{(1)}}{\partial \xi}+B \frac{\partial^{3} \phi^{(1)}}{\partial \xi^{3}}-\frac{\eta_{0}}{2} \frac{\partial^{2} \phi^{(1)}}{\partial \xi^{2}}=0
$$

where, the coefficients of $\mathrm{KdVB}$ equation read as

$$
\begin{aligned}
& A=\frac{4 \beta(1-\beta)^{2}-H^{2}(1+\beta)}{4(1+\beta) \sqrt{\beta}}, \\
& B=\frac{6+4 \beta+6 \beta^{2}}{(1-\beta)\left[4(1-\beta)^{2} \beta-H^{2}(1+\beta)\right]} .
\end{aligned}
$$

The analytical exact stationary solution of KdVB equation (Equation (16)), employing "tanh" method [35,36], reads as

$$
\begin{aligned}
\phi_{s}^{(1)}=\frac{3 \eta_{0}^{2}}{50 A B}\{ & \frac{1}{2} \operatorname{sech}^{2}\left[\frac{\eta_{0}(\xi-u \tau)}{20 B}\right] \\
+ & {\left.\left[1-\tanh \left[\frac{\eta_{0}(\xi-u \tau)}{20 B}\right]\right]\right\} }
\end{aligned}
$$

Simple evaluation of the coefficients of KdVB equation reveals that the dissipative coefficient solely depends on the normalized kinematic viscosity parameter of plasma and is independent from fractional number-density of pair or quantum diffraction parameter. However, the dispersive term is governed by the fractional pair numberdensity as well as the quantum diffraction parameter. It is further noticed that, the dispersive term vanishes $(B=0)$ at some critical quantum diffraction parameter value, $H_{c r}$.

$$
H_{c r}=2(1-\beta) \sqrt{\frac{\beta}{1+\beta}} .
$$

Only at this critical value of, $H_{c r}$, which is solely determined by the fractional pair number-density, $\beta$, the plasma becomes dispersion-less. In terms of KdVB coefficients and the fractional pair concentration, we may also express the maximum shock-amplitude in the following forms

$$
\begin{aligned}
\phi_{0} & =\frac{3 \eta_{0}{ }^{2}}{25 A B} \\
& =\frac{48 \beta(1-\beta)(1+\beta)^{2}}{25\left[4 \beta(1-\beta)^{2}-H^{2}(1+\beta)\right][3+\beta(2+3 \beta)]} .
\end{aligned}
$$

As it is clearly observed, this value diverges at $H_{c r}$ point. It is also remarked that the shock-wave type can be either compressive or rarefactive depending of the sign of the dispersion coefficient in Equation (27).

On the other hand, the traveling-wave solution of Equation (27) can be obtained solving the governing reduced equation, transformed by $\mu=\xi-u \tau$ and integrated once with appropriate boundary conditions, defined below

$$
-u \phi^{(1)}+A\left(\phi^{(1)}\right)^{2}+B \frac{\partial^{2} \phi^{(1)}}{\partial \mu^{2}}-\frac{\eta_{0}}{2} \frac{\partial \phi^{(1)}}{\partial \mu}=0,
$$

the particular solution [37] of which, in bounded form, reads as

$$
\phi_{t}^{(1)}=\frac{6\left(2 B+5 \eta_{0}\right)}{25 A}-\frac{6 \eta_{0}}{5 A} \tanh \mu-\frac{12 B}{25 A} \tanh ^{2} \mu .
$$

However, it is remarked that in this solution the wave amplitude vanishes as $\mu \rightarrow \infty$, as it should.

\section{Numerical Analysis and Discussions}

In this numerical scheme we may use values given in [38] for a typical white dwarf, namely, $n_{e 0}, n_{p 0} \sim 10^{28} \mathrm{~cm}^{-3}$ and $n_{i 0} \sim 10^{27} \mathrm{~cm}^{-3}$. These values result in parameter $H$ of order of unity. Some other values of $0.5>H \geq 0$ has been employed in [39] for a quantum electron-positron-ion plasma. However, the value of this parameter 
can be even larger for the metallic electrons at room temperature, e.g. in the range of $6 \gtrsim H_{\text {metallic }} \gtrsim 2$ as noted in Ref. [33]. On the other hand, it is noted that for heavier ions as pair the Fermi-temperature may be within few thousandth degrees of kelvins resulting in $H$-values much less [38] than that for ordinary electrons in metals

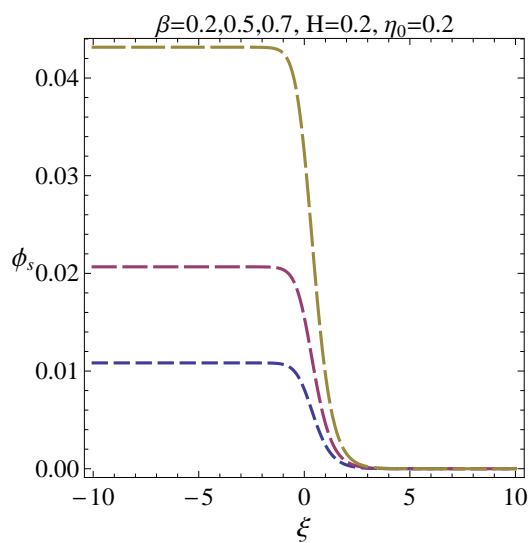

(a)

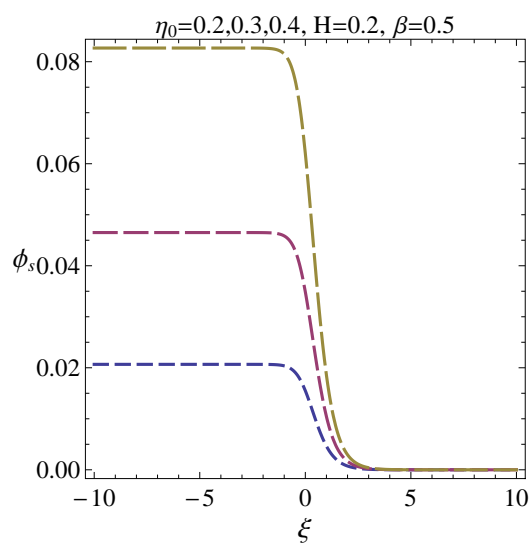

(c)

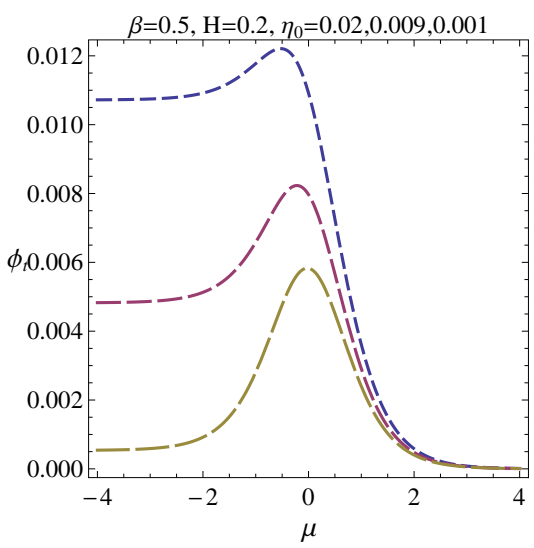

(e)
$(0.01 \gtrsim H \gtrsim 0)$. To base our analysis for a wider range of $H$ parameter we consider its value in the range $1>H>0$ without loss of generality.

Figures 1(a) and 1(b) shows the shock-wave (compressive and rarefactive) profiles for different values of fractional positive to negative pair-concentration, $\beta$

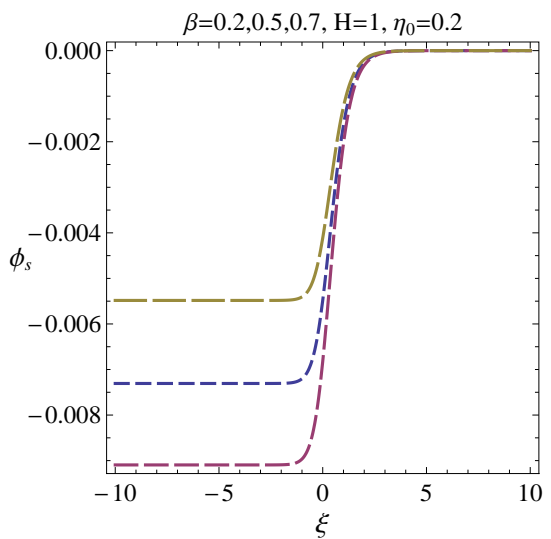

(b)

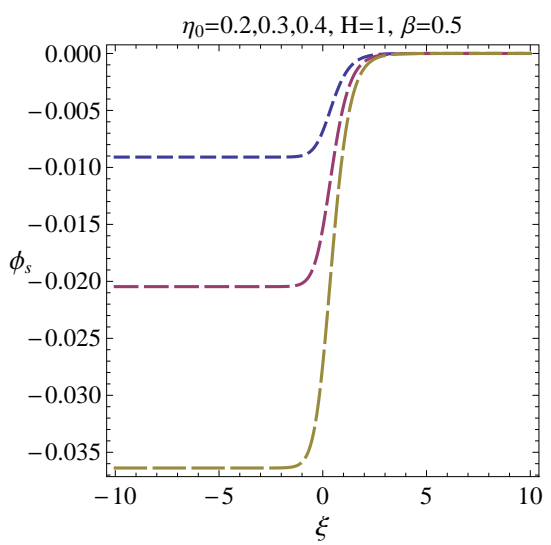

(d)

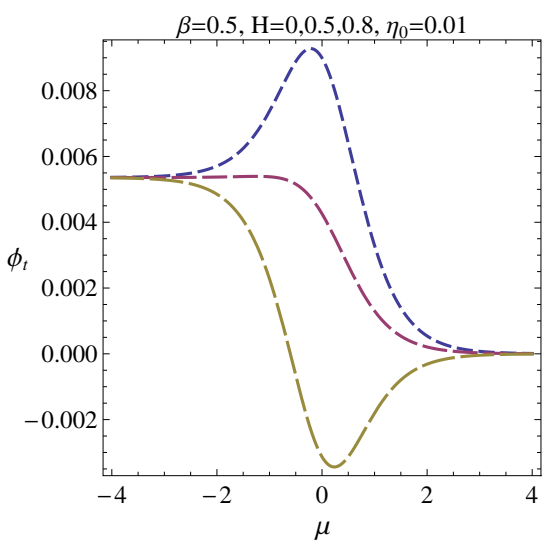

(f)

Figure 1. Depicts the profiles and variations of compressive and rarefactive quantum electrostatic shock-wave amplitudes with respect to fractional positive to negative particle concentration, $\beta$, quantum diffraction parameter, $H$ and scaled plasma kinematic viscosity, $\eta_{0}$ in stationary (Figures 1(a)-1(d)) and traveling wave (Figures 1(e) and 1(f)) frames. The dash sizes in each plot are arranged to appropriately represent the relative values of varied parameter. 
and for two fixed value of quantum diffraction parameter, $H$. As it is clear, the decrease in relative concentration of positive inertiall-less particles leads to an increase in the shock-wave hight for $H=0.2$ (compressive) in the stationary-wave reference frame. However, for $H=1$ (rarefactive) the shock hight first increases and then decreases with decrease of fractional positive to negative pair-concentration, $\beta$, for a fixed value of normalized plasma viscosity, $\eta_{0}$. Also, Figures 1(c) and 1(d) reveal that the shock-wave hight increases as the normalized kinematic-viscosity increases (for both compressive and rarefactive shock profiles) in the stationary-wave reference frame for a fixed value of $\beta$, regardless of the value of $H$.

On the other hand, Figures 1(e) and 1(f) show the shock-wave profiles in the traveling-wave frame. It is evident that with decrease in the value of normalized kinematic-viscosity, $\eta_{0}$, the shock profile transforms

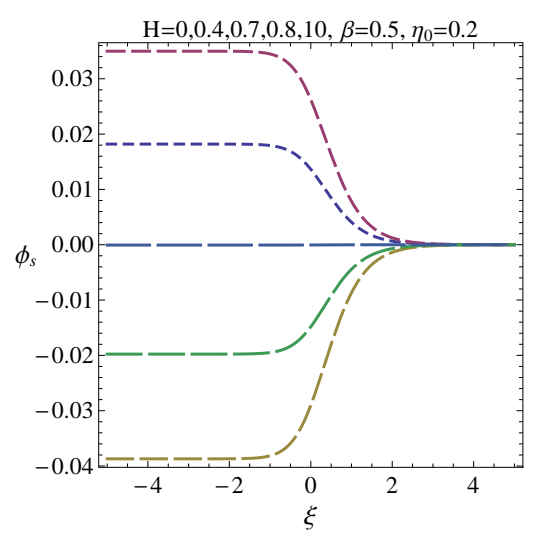

(a)

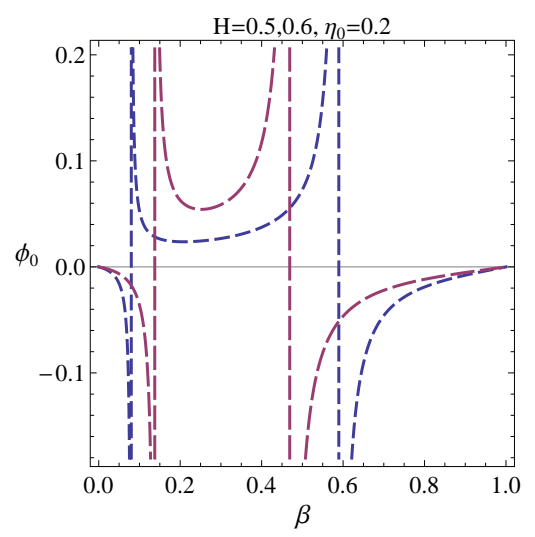

(c) into the bell-shaped soliton profile (Figure 1(e)). This is very similar to ion-acoustics shock behavior reported for electron-positron-ion plasma [40]. Furthermore, Figure $\mathbf{1 ( f )}$ indicates the effect of quantum diffraction parameter, $H$, on the wave profile, where its increase changes the wave profile from compressive bell-shape soliton to shock-wave and then further increase changes the shock profile into a rarefactive bell-shape soliton, leaving the initial wave-hight unchanged.

As it was mentioned in previous section there exist a critical $H$ value for which the amplitude of stationary shock-wave solution diverges, when the dispersion coefficient in Equation (16) vanishes. For values below this critical quantum diffraction parameter, as it is observed from Figure 2(a) the shock-wave shapes are compressive and rarefactive otherwise. It is further observed from Figure 2(a) that for a fixed value of relative dust concentration and normalized kinematic-viscosity the shock

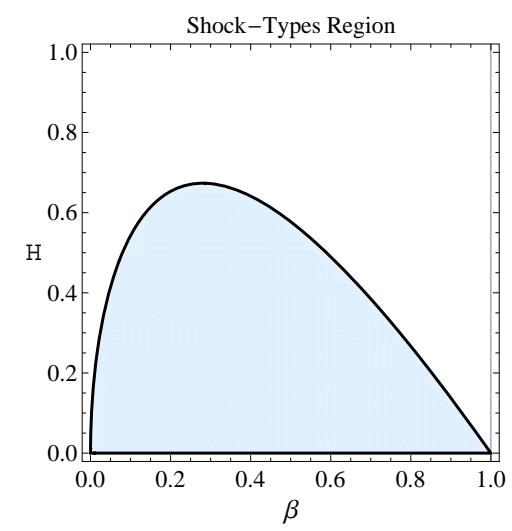

(b)

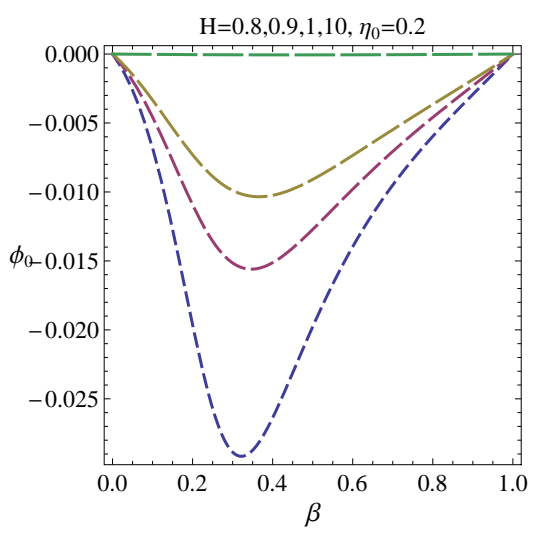

(d)

Figure 2. (a) Shows the variations of compressive and rarefactive quantum electrostatic shock-wave amplitudes in stationary frame with respect to quantum diffraction parameter $\boldsymbol{H}$ for fixed values of scaled plasma viscosity, $\eta_{0}$ and fractional positive to negative particle concentration, $\beta$; (b) Depicts the compressive (grey) and rarefactive (white) shock-wave occurrence regions in $H-\beta$ plane (in stationary frame); (c) Shows the transitions of shock wave profile from rarefactive to compressive and viceversa in stationary frame for values of quantum diffraction parameter below the critical value, $H_{c r}$; (d) Shows the variation rarefactive shock-wave amplitude in stationary frame with respect to relative concentration of dust for different values of quantum diffraction parameter $H$ above critical value $\left(H_{c r}\right)$ and fixed normalized plasma viscosity. The dash sizes in each plot are arranged to represent the values of varied parameter, appropriately. 
wave-hight increase (decreases) with increase of the quantum diffraction parameter value, $H$, for compressive (rarefactive) shock profile. For very high values of quantum diffraction parameters, however, the shock-wave is washed-out ( $H=10$ in Figure 2(a) and Figure 2(d)).

Figure 2(b) shows the grey (white) region in $H-\beta$ plane for which the shock profile is compressive (rarefactive). It is noted that bellow the maximum value of $H_{\text {cr-max }} \approx 0.67$ which corresponds to the value of $\beta=0.28$, for every given fixed nonzero value of quantum diffraction parameter by going from $\beta=0$ to $\beta=1$ two critical points for $\beta$ are encountered for which the shock-wave amplitude becomes infinite and consequently the shape of shock profile is changed from either compressive to rarefactive or viceversa. This feature is more clearly depicted in Figures 2(c) and 2(d) for different values of $H$. By approaching these critical $\beta$-values (Equation (20)) from either sides one observes that shock-wave become more steep and it becomes higher in altitude.

On the other hand, for values of quantum diffraction parameters larger than the critical-value $(H>0.67)$, the shock-wave profile is always rarefactive amplitude of which increases with increase of fractional positive to negative concentration up to a maximum value and then decreases by further decrease in fractional positive to negative pair-concentration. Therefore, it is generally concluded that, the type of shock profile is determined solely by the values of quantum diffraction parameter and the fractional positive to negative pair-concentration, $\beta$, for $0.67>H$ and is always rarefactive for $H>0.67$, in the stationary shock-wave.

\section{Conclusion}

The small- but finite-amplitude quantum shock-wave propagation was studied in the framework of quantum hydrodynamics model using reductive perturbation method in both stationary and traveling-wave reference frames. It was shown that in stationary reference frame a critical quantum diffraction parameter value $\left(H_{c r}\right)$ exists bellow/above which the shock profile is compressive/ rarefactive for fixed values of scaled kinematic-viscosity and dust concentration. It was also found that, in traveling-wave frame the wave profile can change from shock profile to bell-shaped soliton profile with decrease in the value of scaled kinematic-viscosity or it can change from compressive bell-shape to rarefactive bell-shaped soliton profile depending on the value of quantum diffraction parameter.

\section{REFERENCES}

[1] M. Bonitz, D. Semkat, A. Filinov, V. Golubnychyi, D. Kremp, D. O. Gericke, M. S. Murillo, V. Filinov, V. For- tov and W. Hoyer, "Theory and Simulation of Strong Correlations in Quantum Coulomb Systems," Journal of Physics A, Vol. 36, No. 22, 2003, pp. 5921-5930. doi:10.1088/0305-4470/36/22/313

[2] G. Manfredi, "How to Model Quantum Plasmas," Fields Institute Communications, Vol. 46, 2005, pp. 263-287.

[3] A. Markowich, C. Ringhofer and C. Schmeiser, "Semiconductor Equations," Springer, Vienna, 1990.

[4] D. Bohm and D. Pines, "A Collective Description of Electron Interactions: III. Coulomb Interactions in a Degenerate Electron Gas," Physical Review, Vol. 92, No. 3, 1953, pp. 609-625.

[5] D. Pines, "A Collective Description of Electron Interactions: IV. Electron Interaction in Metals," Physical Review, Vol. 92, 1953, p. 609. doi:10.1103/PhysRev.92.626

[6] P. Levine and O. V. Roos, "Plasma Theory of the ManyElectron Atom," Physical Review, Vol. 125, No. 1, 1962, pp. 207-213. doi:10.1103/PhysRev.125.207

[7] H. Haug and S. W. Koch, "Quantum Theory of the Optical and Electronic Properties of Semiconductors," World Scientific, Singapore, 2004.

[8] C. Gardner, "The Quantum Hydrodynamic Model for Semiconductor Devices," SIAM Journal on Applied Mathematics, Vol. 54, No. 2, 1994, pp. 409-427. doi:10.1137/S0036139992240425

[9] L. O. Silva, R. Bingham, J.M. Dawson, J. T. Mendonca and P. K. Shukla, "Neutrino Driven Streaming Instabilities in a Dense Plasma," Physical Review Letters, Vol. 83, No. 14, 1999, pp. 2703-2706. doi:10.1103/PhysRevLett.83.2703

[10] M. Akbari-Moghanjoughi, "Effects of Ion-Temperature on Propagation of the Large-Amplitude Ion-Acoustic Solitons in Degenerate Electron-Positron-Ion Plasmas," Physics of Plasmas, Vol. 17, No. 8, 2010, Article ID: 082315. doi:10.1063/1.3480117

[11] M. Akbari-Moghanjoughi, "Propagation and Head-On Collisions of Ion-Acoustic Solitons in a Thomas-Fermi Magnetoplasma: Relativistic Degeneracy Effects," Physics of Plasmas, Vol. 17, No. 7, 2010. doi: $10.1063 / 1.3449590$

[12] R. Sabry, W. M. Moslem, F. Haas, S. Ali and P. K. Shukla, "Nonlinear Structures: Explosive, Soliton, and Shock in a Quantum Electron-Positron-Ion Magnetoplasma," Physics of Plasmas, Vol. 15, No. 12, 2008, Article ID: 122308 . doi:10.1063/1.3037265

[13] W. Oohara and R. Hatakeyama, "Pair-Ion Plasma Generation Using Fullerenes," Physical Review Letters, Vol. 91, No. 20, 2003, Article ID: 205005.

[14] W. Oohara, D. Date and R. Hatakeyama, "Electrostatic Waves in a Paired Fullerene-Ion Plasma," Physical Review Letters, Vol. 95, No. 10, 2005, Article ID: 175003. doi:10.1103/PhysRevLett.95.175003

[15] W. Oohara and R. Hatakeyama, "Basic Studies of the Generation and Collective Motion of Pair-Ion Plasmas," Physics of Plasmas, Vol. 14, No. 3, 2007, Article ID: 055704. doi:10.1063/1.2436854

[16] W. F. El-Taibany and M. Waidati, "Nonlinear Quantum 
Dust Acoustic Waves in Nonuniform Complex Quantum Dusty Plasma," Physics of Plasmas, Vol. 14, No. 4, 2007, Article ID: 042302. doi:10.1063/1.2717883

[17] M. Marklund, L. Stenflo, P. K. Shukla and G. Brodin, "Quantum Electrodynamical Effects in Dusty Plasmas," Physics of Plasmas, Vol. 12, No. 7, 2005, Article ID: 072111. doi:10.1063/1.1960008

[18] F. Haas, L. G. Garcia, J. Goedert and G. Manfredi, "Quantum Ion-Acoustic Waves," Physics of Plasmas, Vol. 10, No. 10, 2003, p. 3858. doi:10.1063/1.1609446

[19] F. Haas, "A Magnetohydrodynamic Model for Quantum Plasmas," Physics of Plasmas, Vol. 12, No. 6, 2005, Article ID: 062117. doi:10.1063/1.1939947

[20] N. Shukla, P. K. Shukla, G. Brodin and L. Stenflo, "Ion Streaming Instability in a Quantum Dusty Magnetoplasma," Physics of Plasmas, Vol. 15, No. 4, 2008, Article ID: 044503. doi:10.1063/1.2909533

[21] W. Masood and A. Mushtaq, "Electron Acoustic Soliton in a Quantum Magnetoplasma," Physics of Plasmas, Vol. 15, No. 2, 2008, Article ID: 022306. doi:10.1063/1.2841036

[22] P, Chatterjee, K. Roy, S. V. Muniandy and C. S. Wong, "Dressed Soliton in Quantum Dusty Pair-Ion Plasma," Physics of Plasmas, Vol. 16, No. 11, 2009, Article ID: 112106. doi:10.1063/1.3263695

[23] M. Akbari-Moghanjoughi, "Dressed Electrostatic Solitary Waves in Quantum Dusty Pair Plasmas," Physics of Plasmas, Vol. 17, No. 5, 2010, Article ID: 052302. doi:10.1063/1.3392289

[24] M. Akbari-Moghanjoughi, "Propagation of ArbitraryAmplitude Nonlinear Quantum Ion-Acoustic Waves in Electron-Ion Plasmas: Dimensionality Effects," IEEE Transactions on Plasma Science, Vol. 38, No. 12, 2010, pp. 3336-3341. doi:10.1109/TPS.2010.2083700

[25] M. Akbari-Moghanjoughi, "Propagation and Oblique Collision of Electrostatic Solitary Waves in Quantum Pair-Plasmas," Physics of Plasmas, Vol. 17, No. 8, 2010, Article ID: 082317. doi:10.1063/1.3480307

[26] M. Akbari-Moghanjoughi, "Double-Wells and DoubleLayers in Dusty Fermi-Dirac Plasmas: Comparison with the Semiclassical Thomas-Fermi Counterpart," Physics of Plasmas, Vol. 17, No. 12, 2010, Article ID: 123709. doi:10.1063/1.3527997

[27] P. K. Shukla and B. Eliasson, "Nonlinear Instability and Dynamics of Polaritons in Quantum Systems," New Journal of Physics, Vol. 9, No. 98, 2007, p. 98. doi:10.1088/1367-2630/9/4/098

[28] W. Masood, M. Siddiq, S, Nargis and A. M. Mirza, "Propagation and Stability of Quantum Dust-Ion-Acoustic Shock Waves in Planar and Nonplanar Geometry,"
Physics of Plasmas, Vol. 16, No. 1, 2009, Article ID: 013705. doi: $10.1063 / 1.3068171$

[29] S. A. Khan and Q. Haque, "Electrostatic Nonlinear Structures in Dissipative Electron-Positron-Ion Quantum Plasmas," Chinese Physics Letters, Vol. 25, 2008, p. 4329. doi:10.1088/0256-307X/25/12/040

[30] W. Masood, A. M. Mirza and M. Hanif, "Ion Acoustic Shock Waves in Electron-Positron-Ion Quantum Plasma," Physics of Plasmas, Vol. 15, No. 7, 2008, Article ID: 072106. doi:10.1063/1.2949702

[31] A. P. Misra, "Dust Ion-Acoustic Shocks in Quantum Dusty Pair-Ion Plasmas," Physics of Plasmas, Vol. 16, No. 3, 2009, Article ID: 033702. doi:10.1063/1.3085789

[32] A. Shah and R. Saeed, "Ion Acoustic Shock Waves in a Relativistic Electron-Positron-Ion Plasmas," Physical Letters A, Vol. 373, No. 45, 2009, pp. 4164-4168. doi:10.1016/i.physleta.2009.09.028

[33] F. Haas, L. G. Garcia, J. Goedert and G. Manfredi, "Quantum Ion-Acoustic Waves," Physics of Plasmas, Vol. 10, No. 10, 2003, p. 3858. doi:10.1063/1.1609446

[34] H. Washimi and T. Taniuti, "Propagation of Ion-Acoustic Solitary Waves of Small Amplitude," Physical Review Letters, Vol. 17, No. 19, 1966, pp. 996-998. doi:10.1103/PhysRevLett.17.996

[35] W. Malfliet, "Solitary Wave Solutions of Nonlinear Wave Equations," American Journal of Physics, Vol. 60, No. 7, 1992, pp. 650. doi:10.1119/1.17120

[36] W. Malfliet, "The Tanh Method: A Tool for Solving Certain Classes of Nonlinear Evolution and Wave Equations," Journal of Computational and Applied Mathematics, Vol. 164, No. 3, 2004, pp. 529-541. doi:10.1016/S0377-0427(03)00645-9

[37] B. Sahu and R. Roychoudhury, "Travelling Wave Solution of Korteweg-de Vries-Burger's Equation," Czechoslovak Journal of Physics, Vol. 53, No. 6, 2003, pp. 517527. doi:10.1023/A:1024657626565

[38] S. Ali, W. M. Moslem, P. K. Shukla and R. Schlickeiser, "Linear and Nonlinear Ion-Acoustic Waves in an Unmagnetized Electron-Positron-Ion Quantum Plasma," Physics of Plasmas, Vol. 14, No. 8, 2007, Article ID: 082307. doi:10.1063/1.2750649

[39] W. M. Moslem, P. K. Shukla, S. Ali and R. Schlickeiser, "Quantum Dust-Acoustic Double Layers," Physics of Plasmas, Vol. 14, No. 4, 2007, Article ID: 042107. doi:10.1063/1.2719633

[40] R. Saeed and A. Shah, "Nonlinear Korteweg de Vries Burger Equation for Ion Acoustic Shock Waves in a Weakly Relativistic Electron-Positron-Ion Plasma with Thermal Ions," Physics of Plasmas, Vol. 17, No. 3, 2010, Article ID: 032308. doi:10.1063/1.3328805 